\title{
Charge measurement of cosmic rays by Plastic Scintillator Detector of DAMPE
}

\author{
Peng-Xiong Ma ${ }^{a, *}$ M. Di Santo, ${ }^{b, c}$ Zhi-Hui Xu ${ }^{a, d}$ and Yong-Jie Zhang ${ }^{e}$ on behalf of \\ the DAMPE Collaboration \\ (a complete list of authors can be found at the end of the proceedings) \\ ${ }^{a}$ Key Laboratory of Dark Matter and Space Astronomy, Purple Mountain Observatory, Chinese Academy \\ of Sciences, Nanjing 210023, China \\ ${ }^{b}$ Gran Sasso Science Institute (GSSI), Via Iacobucci 2, I-67100 L'Aquila, Italy \\ ${ }^{c}$ Istituto Nazionale di Fisica Nucleare (INFN) -Laboratori Nazionali del Gran Sasso, I-67100 Assergi, \\ L'Aquila, Italy \\ ${ }^{d}$ School of Astronomy and Space Science, University of Science and Technology of China, Hefei 230026, \\ China \\ ${ }^{e}$ Institute of Modern Physics, Chinese Academy of Sciences, Nanchang Road 509, Lanzhou 730000, China \\ E-mail: mapx@pmo.ac.cn
}

Plastic Scintillator Detector (PSD) of DArk Matter Particle Explorer (DAMPE) is designed to measure the charge of cosmic-rays and it servers as a veto for gamma-rays. In this work, we present some updated correction methods to further improve the quality of PSD charge measurement, especially for heavy nuclei. DAMPE has collected nearly 10 billions events by middle of 2021 , it has substantial potential to measure the spectra of cosmic ray nuclei up to hundreds of TeV energies. These measurements could largely benefit from the correction of the PSD signal.

$37^{\text {th }}$ International Cosmic Ray Conference (ICRC 2021)

July 12th - 23rd, 2021

Online - Berlin, Germany

\footnotetext{
${ }^{*}$ Presenter
} 


\section{Introduction}

The DArk Matter Particle Explorer (DAMPE; [1]) is a satellite-borne cosmic ray particle and $\gamma$-ray detector launched on December 17, 2015. It consists of a Plastic Scintillator Detector (PSD) [2] for charge measurement, a Silicon Tungsten tracKer-converter (STK) [3] for trajectory measurement and $\gamma$-ray to \pm conversion, a $\mathrm{Bi}_{3} \mathrm{Ge}_{4} \mathrm{O}_{12}$ electromagnetic calorimeter (BGO) [4] for energy measurement and electron-hadron discrimination, and a NeUtron Detector (NUD) [5] for enhancement of electron-hadron discrimination. DAMPE is expected to significantly improve the measurement precision of galactic cosmic ray (GCR) spectra up to $100 \mathrm{TeV}$ energies, due to its large acceptance and good energy resolution ( $\sim 1.5 \%$ for electrons and $\gamma$-rays [6] and $\sim 30 \%$ for nuclei $[7,8])$. Dedicated calibrations of each sub-detector show that the instrument works very stably on-orbit [9-11]. In this proceedings, we will present a latest calibration of PSD in order to enhance the quality of charge measurement as a basis for analysis of heavy nuclei spectra with DAMPE.

PSD is composed of two orthogonal planes, with 41 units in each plane. Left panel of Fig. 1 shows the structure of PSD, the red elements are detection units. Each PSD unit is coupled with two photomultiplier tubes (PMT) at each end, respectively, which allows us to measure the charge of incident particle at both ends independently, thus to obtain much better resolution for charge measurements $[2,12]$.
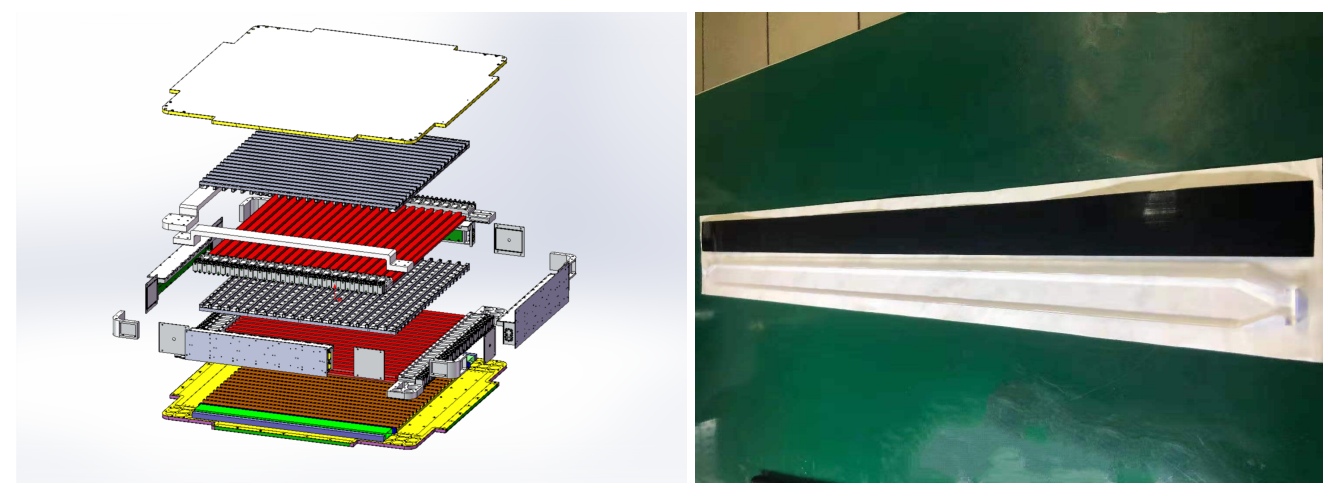

Figure 1: PSD detector. Left side shows the structure of PSD, two planes are orthogonal with each other (red parts); Right picture contains one PSD detection unit with Tyvek 1056D (white) for enhancement of light reflection and heat-shrinkable tube (black) for light shielding.

Many correction and calibration methods have been applied to PSD units in order to improve the resolution of charge measurement. We quote light attenuation correction, geometrical alignment, and quenching-equalization [10, 12, 13]. All these corrections contributed to optimal charge resolution for proton and helium spectra measurement [7, 8] and were based on light nuclei. DAMPE collected more 10 billions of high energy cosmic-rays until now, this huge sample enables us to calibrate the detector also for heavy nuclei.

\section{Method}

\subsection{PSD Charge Reconstruction}

The principle of charge measurement by means of PSD is based on the Bethe-Bloch formula, which indicates the ionization energy within PSD is proportional to the square of charge of particle 
and path length within PSD unit, e.g. $-\Delta E \propto Z^{2} \times L$, where - means energy loss for particle. So charge of particle could be derived from the ionization energy and path length, the former is calculated by the output of PMT at each end, the latter is figured out with combination of the incident trajectory constructed by STK sub-detector and PSD geometry in DAMPE frame.

$\Delta E$ means the ionization energy within PSD unit, which is determined from the output of PMT at each end. In the process of PSD charge reconstruction, we also employed light attenuation correction to reduce the bias came from different hit position [12, 13], alignment of PSD units are also indeed so as to obtain reliable path length [10]. According to simulation, for vertical incident proton, its energy loss in $10 \mathrm{~mm}$ is $2 \mathrm{MeV}$, for isotropic GCR, we need convert its energy loss for any path length into equivalently vertical energy loss, Finally, PSD charge is determined by $Z=\sqrt{\frac{\Delta E \times 10 \mathrm{~mm}}{L \times 2 \mathrm{MeV}}}$.

\subsection{The Phenomenological Method}

One of DAMPE main scientific goals is the precise measurement of GCR spectra from proton to nickel. In order to distinguish the cosmic-ray nuclei with different charge number, a high charge resolution detector is needed. We observed that heavy nuclei show different patterns of PSD signal as a function of hit position along PSD detection units, as shown in Fig. 2. These patterns are observed for each PSD unit, and patterns are different from bar to bar. This feature is due to that light reflection material Tyvek paper did not attach to the surface of scintillator bar uniformly, rather there are tiny wrinkles. The wrinkles would change the light reflection rate depending on the gap distance between the reflection layer and scintillator surface. As shown in Fig. 1, the pattern is same for all measured charges.
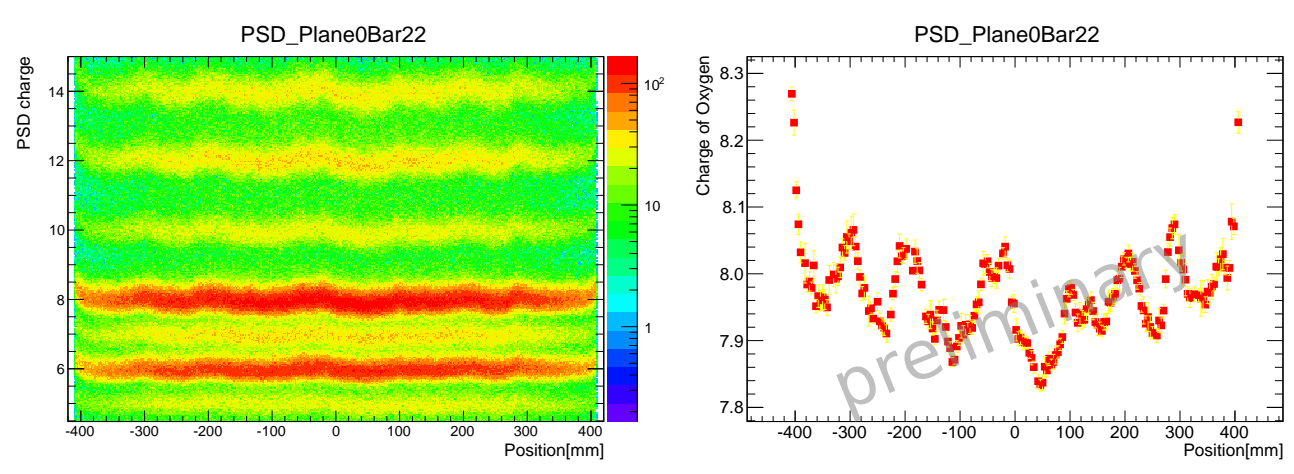

Figure 2: Charge value as function of hit position. Left panel shows the pattern of 23rd PSD unit located in first plane. In the right panel the most probable value (MPV) of the Landau fit convoluted with Gaussian function of PSD signal for oxygen nuclei is shown as a function of hit position.

We developed an empirical method to remove the PSD signal variation with hit position. The method is based on the template-interpolation like that in the right panel of Fig. 2. The pattern of oxygen component acts as template for interpolation to determine a correction factor, and then we apply this factor to all charges reconstructed by this very PSD unit, as shown in Eqa. 1.

$$
Z_{\mathrm{psd}, \text { after correction }}=\frac{8}{Z(\text { hit position })_{\mathrm{O}, \text { template }}} \times Z_{\text {before correction }}
$$



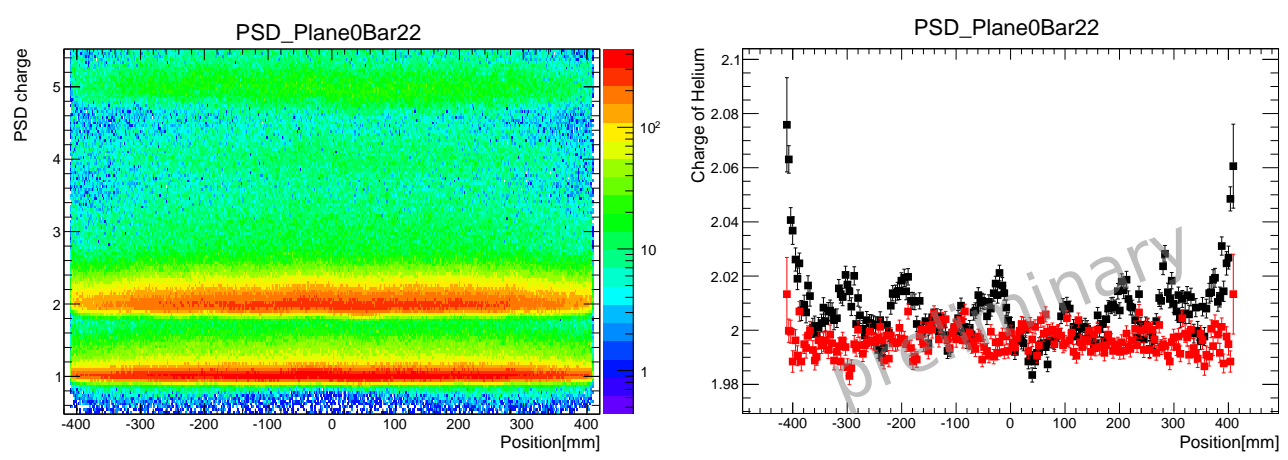

Figure 3: Same as Fig. 2. Left panel shows the pattern of light nuclei of 23rd PSD unit located in first plane. In the right panel the MPV of helium nuclei are shown as a function of hit position before (black squares) and after (red squares) correction, respectively.

where the $Z$ (hit position $)_{\mathrm{O} \text {,template }}$ is obtained from right panel of Fig. 2 with interpolation, the procedure is repeated for all units. In this correction, we applied the oxygen candidates as the template due to the statistics of measured oxygen are relatively dominant among heavy nuclei under the high energy trigger setting [14]. If needed, when DAMPE collects adequate more heavier nuclei in the future, for example iron, we could also update this correction with iron in case of the non-linearity of charge measurement for light and heavy components.

Light components of GCR are majority, Helium of which is second dominant particles among all species of cosmic rays, it has a relative charge resolution better than $\frac{\Delta Z}{Z} \approx 0.5 \%$ in single PSD unit, meanwhile, the non-uniformities of helium among different PSD units are negligible [13], the variation patterns appeared in different units just weak a little the helium resolution, we found that the position correction for helium is not significant in Fig. 3 and 6.
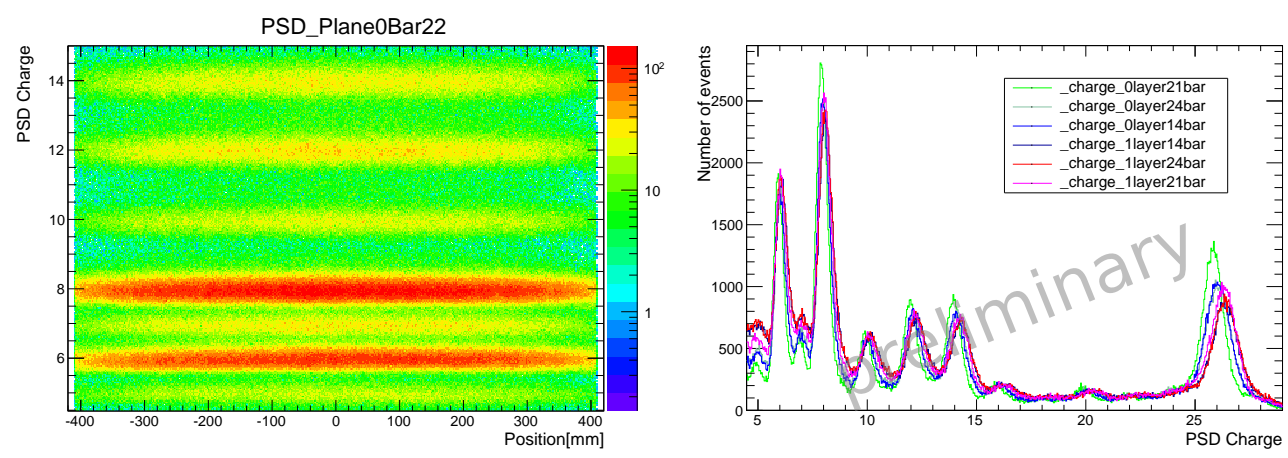

Figure 4: MPV charge values for heavy nuclei versus hit position after correction. Left panel shows the uniformity of charge measurement of the 23rd unit in the first plane. On the right the differences among different PSD units are shown.

The left panel of Fig. 4 shows the relation between charge value and hit position after the correction. As expected, the peculiar pattern visible in left panel of Fig. 2, essentially disappears. The final charge value is determined by at least 4 measurements or at most 8 measurements thanks to the PSD overlapping design. So charge measurement with multi PSD units would give a better resolution. The right side of Fig. 4 presents the PSD charge signal from boron to nickel for different 
units. Small differences of peak position among different PSD units are present. It is trivial to explain this difference as a consequence of the former quenching-equalization based on a sample without position correction introduced in this work. So an additional correction to uniform the charge peak must be applied [13]. Taking into account that DAMPE focus mainly on GCR spectra beyond the geomagnetic cutoff, and the ionization energy deposited in PSD by different particle species shows different pattern, we have to set an energy threshold to uniform charge peaks to obtain a better charge resolution and a precise spectrum measurement at the same time.

Fig. 5 shows charge measurement for single PSD units, the uniformities are obviously improved compared with right plot in Fig. 4. This result lays a solid foundation for precise measurement of cosmic-rays spectra.

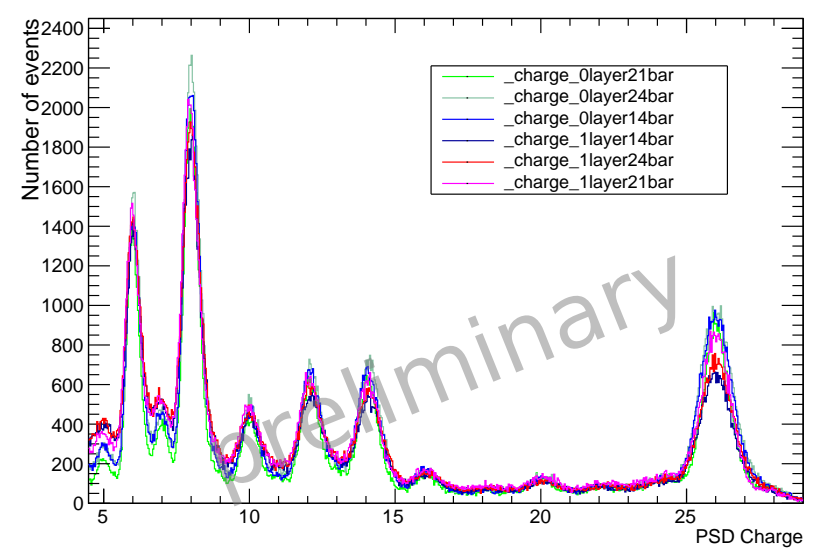

Figure 5: Charge measurement from boron to nickel for several PSD units. The uniformity among units is significant.

\section{Results and summary}

Based on a series of previous works, we added a new correction to eliminate charge fluctuations depending on hit position. Thanks to the application of this new correction, we get uniform results for all PSD units and improve the charge resolution.

In Fig. 6 the charge measurement and its resolution before and after the correction have been compared. The right plot shows charge resolution for helium, carbon and iron as a function of energy deposition within BGO. Black and red filled squares indicate the results after and before the correction presented in this work, respectively. Particularly, for carbon and iron, the resolution improved significantly, which is quite helpful for identifying the heavy cosmic-ray elements.

\section{Acknowledgement}

The DAMPE mission was funded by the strategic priority science and technology projects in space science of Chinese Academy of Sciences. In China the data analysis is supported by the National Key Research and Development Program of China (No. 2016YFA0400200), the National 

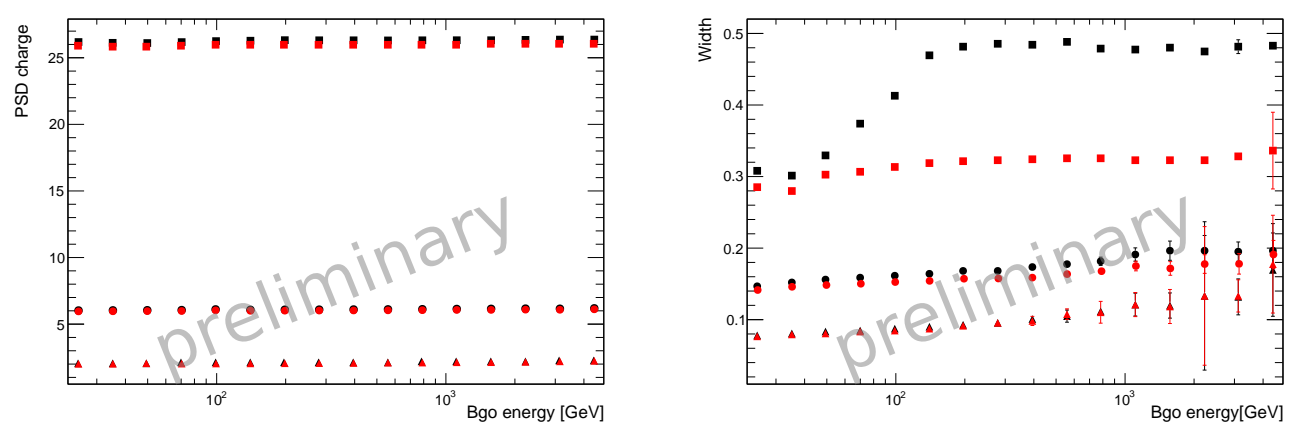

Figure 6: Parameters of Landau+Gaussian fit for PSD charge as a function of energy deposition within BGO (parameters for helium, carbon and iron are presented from below to above on the vertical axis). Left panel shows the charge MPV. Right panel shows the width of charge measurement. Black squares indicate the fit parameters without corrections, red squares correspond to results after applying the correction presented in this work.

Natural Science Foundation of China (Nos. 11921003, 11622327, 11722328, 11851305, U1738205, U1738206, U1738207, U1738208, U1738127), the 100 Talents Program of Chinese Academy of Sciences, the Young Elite Scientists Sponsorship Program by CAST (No. YESS20160196), and the Program for Innovative Talents and Entrepreneur in Jiangsu. In Europe the activities and data analysis are supported by the Swiss National Science Foundation (SNSF), Switzerland, the National Institute for Nuclear Physics (INFN), Italy.

\section{References}

[1] J. Chang et al. (DAMPE Collaboration), Astropart. Phys. 95, 6 (2017).

[2] Y. H. Yu et al., Astropart. Phys. 94, 1 (2017).

[3] P. Azzarello et al., Nucl. Instrum. Meth. A 831, 378 (2016).

[4] Z. Zhang et al., Nucl. Instrum. Meth. A 836, 98 (2016).

[5] Y. Y. Huang et al., Res. Astron. Astrophys. 20, 153 (2020).

[6] G. Ambrosi et al. (DAMPE Collaboration), Nature 552, 63 (2017).

[7] Q. An et al. (DAMPE Collaboration), Science Advances 5, eaax3793 (2019).

[8] F. Alemanno et al. (DAMPE Collaboration), Phys. Rev. Lett. 126, 201102 (2021).

[9] G. Ambrosi et al. (DAMPE Collaboration), Astropart. Phys. 106, 18 (2019).

[10] P. X. Ma et al., Res. Astron. Astrophys. 19, 082 (2019).

[11] A. Tykhonov et al., Nucl. Instrum. Meth. A 924, 309 (2019).

[12] M. Ding et al., Res. Astron. Astrophys. 19, 47 (2019). 
[13] T. K. Dong et al., Astropart. Phys. 105, 31 (2019).

[14] Y. Q. Zhang et al., Res. Astron. Astrophys. 19, 123 (2019). 


\section{Full Authors List: DAMPE Collaboration}

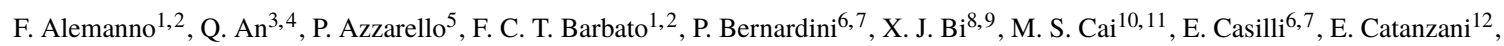
J. Chang ${ }^{10,11}$, D. Y. Chen ${ }^{10}$, J. L. Chen ${ }^{13}$, Z. F. Chen ${ }^{10,11}$, M. Y. Cui ${ }^{10}$, T. S. Cui ${ }^{14}$, Y. X. Cui ${ }^{10,11}$, H. T. Dai ${ }^{3,4}$, A. De Benedittis ${ }^{6,7}$,

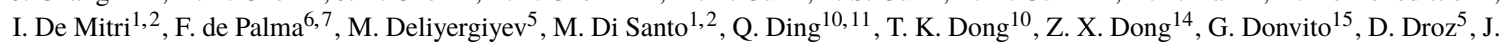
L. Duan ${ }^{13}$, K. K. Duan ${ }^{10}$, D. D’Urso ${ }^{12,1}$, R. R. Fan ${ }^{8}$, Y. Z. Fan ${ }^{10,11}$, K. Fang ${ }^{8}$, F. Fang ${ }^{13}$, C. Q. Feng ${ }^{3,4}$, L. Feng ${ }^{10}$, P. Fusco ${ }^{15,16}$, M. $\mathrm{Gao}^{8}$, F. Gargano ${ }^{15}$, K. Gong ${ }^{8}$, Y. Z. Gong ${ }^{10}$, D. Y. Guo ${ }^{8}$, J. H. Guo ${ }^{10,11}$, S. X. $\mathrm{Han}^{14}$, Y. M. Hu ${ }^{10}$, G. S. Huang ${ }^{3,4}$, X. Y. Huang ${ }^{10,11}$,

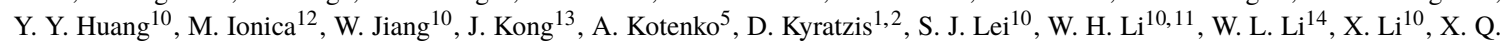

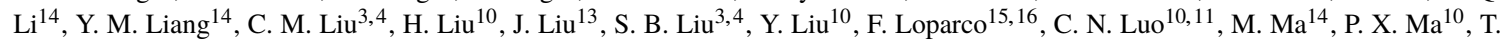
$\mathrm{Ma}^{10}$, X. Y. Ma ${ }^{14}$, G. Marsella ${ }^{6,7}{ }_{2}$, M. N. Mazziotta ${ }^{15}$, D. $\mathrm{Mo}^{13}$, X. Y. Niu ${ }^{13}$, X. Pan ${ }^{10,11}$, A. Parenti ${ }^{1,2}$, W. X. Peng ${ }^{8}$, X. Y. Peng ${ }^{10}$, C. Perrina $^{5,3}$, R. Qiao $^{8}$, J. N. Rao ${ }^{14}$, A. Ruina ${ }^{5}$, M. M. Salinas ${ }^{5}$, G. Z. Shang ${ }^{14}$, W. H. Shen ${ }^{14}$, Z. Q. Shen ${ }^{10}$, Z. T. Shen ${ }^{3,4}$, L. Silveri ${ }^{1,2}$, J. X. Song ${ }^{14}$, M. Stolpovskiy ${ }^{5}$, H. Su ${ }^{13}$, M. Su ${ }^{17}$, H. R. Sun ${ }^{3,4}$, Z. Y. Sun ${ }^{13}$, A. Surdo ${ }^{7}$, X. J. Teng ${ }^{14}$, A. Tykhonov ${ }^{5}$, H. Wang ${ }^{14}$, J. Z.

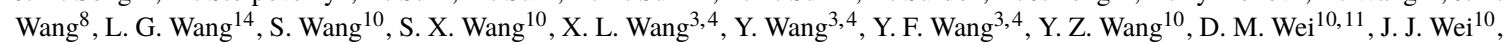
Y. F. $\mathrm{We}^{3,4}$, D. $\mathrm{Wu}^{8}$, J. Wu ${ }^{10,11}$, L. B. $\mathrm{Wu}^{1,2}$, S. S. $\mathrm{Wu}^{14}$, X. Wu $\mathrm{Wu}^{5}$ Z. Q. Xia ${ }^{10}$, H. T. Xu ${ }^{14}$, Z. H. Xu ${ }^{10,11}$, Z. L. Xu ${ }^{10}$, E. H. Xu $u^{3,4}, Z$. Z. Xu ${ }^{3,4}$, G. F. Xue ${ }^{14}$, H. B. Yang ${ }^{13}$, P. Yang ${ }^{13}$, Y. Q. Yang ${ }^{13}$, H. J. Yao ${ }^{13}$, Y. H. Yu ${ }^{13}$, G. W. Yuan ${ }^{10,11}$, Q. Yuan ${ }^{10,11}$, C. Yue ${ }^{10}$, J. J. Zang $^{104}$ S. X. Zhang ${ }^{13}$, W. Z. Zhang ${ }^{14}$, Yan Zhang ${ }^{10}$, Yi Zhang ${ }^{10,11}$, Y. J. Zhang ${ }^{13}$, Y. L. Zhang ${ }^{3,4}$, Y. P. Zhang ${ }^{13}$, Y. Q. Zhang ${ }^{10}$, Z. Zhang ${ }^{10}$, Z. Y. Zhang ${ }^{3,4}$, C. Zhao ${ }^{3,4}$, H. Y. Zhao ${ }^{13}$, X. F. Zhao ${ }^{14}$, C. Y. Zhou ${ }^{14}$, and Y. Zhu ${ }^{14}$

${ }^{1}$ Gran Sasso Science Institute (GSSI), Via Iacobucci 2, I-67100 L'Aquila, Italy

${ }^{2}$ Istituto Nazionale di Fisica Nucleare (INFN) -Laboratori Nazionali del Gran Sasso, I-67100 Assergi, L'Aquila, Italy

${ }^{3}$ State Key Laboratory of Particle Detection and Electronics, University of Science and Technology of China, Hefei 230026, China

${ }^{4}$ Department of Modern Physics, University of Science and Technology of China, Hefei 230026, China

${ }^{5}$ Department of Nuclear and Particle Physics, University of Geneva, CH-1211, Switzerland

${ }^{6}$ Dipartimento di Matematica e Fisica E. De Giorgi, Universita‘ del Salento, I-73100, Lecce, Italy

${ }^{7}$ Istituto Nazionale di Fisica Nucleare (INFN) - Sezione di Lecce, I-73100, Lecce, Italy

${ }^{8}$ Institute of High Energy Physics, Chinese Academy of Sciences, Yuquan Road 19B, Beijing 100049, China

${ }^{9}$ University of Chinese Academy of Sciences, Yuquan Road 19A, Beijing 100049, China

${ }^{10}$ Key Laboratory of Dark Matter and Space Astronomy, Purple Mountain Observatory, Chinese Academy of Sciences, Nanjing 210023, China

${ }^{11}$ School of Astronomy and Space Science, University of Science and Technology of China, Hefei 230026, China

${ }^{12}$ Istituto Nazionale di Fisica Nucleare (INFN) - Sezione di Perugia, I-06123 Perugia, Italy

${ }^{13}$ Institute of Modern Physics, Chinese Academy of Sciences, Nanchang Road 509, Lanzhou 730000, China

${ }^{14}$ National Space Science Center, Chinese Academy of Sciences, Nanertiao 1, Zhongguancun, Haidian district, Beijing 100190, China

${ }^{15}$ Istituto Nazionale di Fisica Nucleare (INFN) - Sezione di Bari, I-70125, Bari, Italy

${ }^{16}$ Dipartimento di Fisica "M. Merlin" dell'Universita“ e del Politecnico di Bari, I-70126, Bari, Italy

${ }^{17}$ Department of Physics and Laboratory for Space Research, the University of Hong Kong, Pok Fu Lam, Hong Kong SAR, China

${ }^{1}$ Now at Dipartimento di Chimica e Farmacia, Universita‘ di Sassari, I-07100, Sassari, Italy.

${ }^{2}$ Now at Dipartimento di Fisica e Chimica "E. Segre", Universita“ degli Studi di Palermo, via delle Scienze ed. 17, I-90128 Palermo, Italy.

${ }^{3}$ Also at Institute of Physics, Ecole Polytechnique Federale de Lausanne (EPFL), CH-1015 Lausanne, Switzerland.

${ }^{4}$ Also at School of Physics and Electronic Engineering, Linyi University, Linyi 276000, China. 